\title{
Hybrid opto-mechanical systems with nitrogen-vacancy centers
}

\author{
YIN ZhangQi ${ }^{1 *}$, ZHAO Nan ${ }^{2,3 \dagger} \&$ LI TongCang ${ }^{4,5 \ddagger}$ \\ ${ }^{1}$ Center for Quantum Information, Institute for Interdisciplinary Information Sciences, Tsinghua University, Beijing 100084, China; \\ ${ }^{2}$ Beijing Computational Science Research Center, Beijing 100084, China; \\ ${ }^{3}$ Synergetic Innovation Center of Quantum Information and Quantum Physics, University of Science and Technology of China, Hefei 230026, China; \\ ${ }^{4}$ Department of Physics and Astronomy and School of Electrical and Computer Engineering, Purdue University, West Lafayette, IN 47907, USA; \\ ${ }^{5}$ Birck Nanotechnology Center, Purdue University, West Lafayette, IN 47907, USA
}

Received Month date, Year; accepted Month date, Year

\begin{abstract}
In this review, we briefly overview recent works on hybrid (nano) opto-mechanical systems that contain both mechanical oscillators and diamond nitrogen-vacancy (NV) centers. We review two different types of mechanical oscillators. The first one is a clamped mechanical oscillator, such as a cantilever, with a fixed frequency. The second one is an optically trapped nano-diamond with a build-in nitrogen-vacancy center. By coupling mechanical resonators with electron spins, we can use the spins to control the motion of mechanical oscillators. For the first setup, we discuss two different coupling mechanisms which are magnetic coupling and strain induced coupling. We summarize their applications such as cooling the mechanical oscillator, generating entanglements between NV centers, squeezing spin ensembles and et al. For the second setup, we discuss how to generate quantum superposition states with magnetic coupling, and realize matter wave interferometer. We will also review its applications as ultra-sensitive mass spectrometer. Finally, we discuss new coupling mechanisms and applications of the field.
\end{abstract}

Opto-mechanics, Nitrogen-vacancy center, Hybrid system

PACS number(s): ??????

Citation: $\quad$ ???, et al. ???. Sci China-Phys Mech Astron, 2014, 57: 1-6, doi:

\section{Introduction}

In 2012, David Wineland, together with Serge Haroche, won Nobel prize "for ground-breaking experimental methods that enable measuring and manipulation of individual quantum systems" [1]. In his lab, the motion and the electron spin of trapped ions couple with each other. Therefore, the motional degree of freedom of the trapped ions can be coherently manipulated, such as the ground state cooling, Fock states generating and detecting with unit fidelity and efficiency [2]. Using these technologies, David Wineland generated Shrödinger's cat states with trapped ions [3,4], which for many years only existed in through experiment.

*Corresponding author (email: yinzhangqi@ mail.tsinghua.edu.cn)

†Corresponding author (email: nzhao@csrc.ac.cn)

†Corresponding author (email: tcli@purdue.edu)
The "true" Shrödinger's cat state is defined by a microscopic two-level system entangling with a macroscopic system. New technology, such as opto-mechanics, is needed to generate quantum superpositions in macroscopic systems. Opto-mechanics studies the radiation pressure on the mechanical oscillator, and how to use this pressure to manipulate the motion of mechanical systems. In 17th century Kepler found the radiation pressure of light when he studied the comet tails. 1n 1909, Einstein studied the statistics of radiation pressure force fluctuations on movable mirror [5]. In 1960s, Braginsky studied the role of radiation pressure in the context of interferometers, and proposed to cool the motionnal temperature of the mirror by radiation pressure [6]. In 
1969, A. Ashkin observed optical trapping of micron-sized particles in liquid [7]. Two years later, optical levitation of glass spheres by an upward-propagating laser beam in both air and vacuum was demonstrated [8]. The optical tweezers now is widely used in atomic physics, chemistry and biology.

Recent years, we witness great developments of optomechanics [9--11]. We have achieved the quantum ground state of macroscopic mechanical oscillators by traditional cryogenic techniques in 2010 [12], or by active cooling in 2011 [13, 14]. We have developed many technologies based on opto-mechanics. For example, we have transferred the signals from light to mechanical oscillation, and vise versa [15]. We have realized optomechanically induced transparency [16] and generated squeezed lights in opto-mechanical systems [17]. By using mechanical oscillator as an interface, quantum information has been able to exchange between microwave superconducting circuits and optical lights [18-20].

In future we aim to achieve the similar controlling ability of macroscopic resonators as we did in the trapped ions. Then we can generate Schrödinger's cat states with macroscopic objects [21], or even macroscopic living objects, such as virus [22]. As macroscopic quantum superposition states become bigger and bigger, we hope to identify the intrinsic decoherence mechanisms such as gravity induced decoherence [23], Continuous Spontaneous Localization models [24 25], and et al.. For readers who are interested in the theory and developments of optomechanics, please read these reviews [9-11].

In order to generate the non-classical states in an optomechanical oscillator, strongly quadratic coupling between the oscillator and the optical mode is essential [21]. However, it is usually very challenging to get the large quadratic coupling in experiments. Inspired by the trapped ion experiments, the quadratic coupling can be replaced by coupling the mechanical oscillator to a two-level quantum system, which can be controlled well externally. In order to get the stable macroscopic quantum states, the coherence time of both the mechanical oscillator and the spin $1 / 2$ should be maximized. There are several proposals that based on quantum dots [26, 27], atoms [28], superconducting qubits [12] and Nitrogen-vacancy centers (NV centers) [29-32]. Among all these proposals, the ones with NV centers are most attractive. As NV centers have presented the coherence even at room temperature.

NV centers in diamond are usually regarded as artificial atoms in solid systems. The diamond lattice consists of covalently bond carbon atoms, which makes diamond very stiff. The valence electrons in diamond have huge bandgap (5.48 ev), which makes it transparent deep into the UV. In its lattice, nitrogen and vacancy are the most common defects. As shown in Fig. 11 an NV center consists of a nitrogen atom and a vacancy at the nearest neighboring site. The NV centers are usually negatively charged, possessing 6 electrons and spin $S=1$ in the ground state.
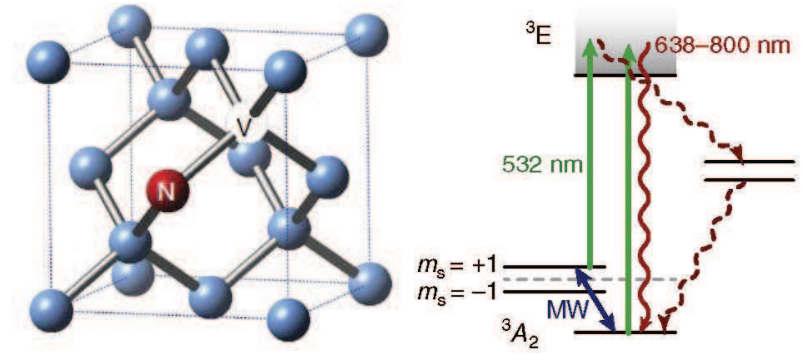

Figure 1 Left figure shows lattice structure of diamond with nitrogenvacancy center. Right one shows the negatively charged NV center electronic energy level structure. Electronic spin polarization and readout is performed by optical excitation at $532 \mathrm{~nm}$ and red fluorescence detection around 637 $\mathrm{nm}$. Ground-state spin manipulation is achieved by resonant microwave excitation. The ground-state triplet has a zero magnetic field splitting $\simeq 2.87$ GHz. Figure adopted from Ref. [33]. Copyright (2012) by Nature Publishing Group.

NV centers are promising candidate system for quantum information processing. Single NV centers can be addressed using the confocal microscopy technique. The spin state of $\mathrm{NV}$ centers can be initialized and read-out at room temperature, respectively, by optical pumping and spin-dependent fluorescence. Furthermore, the spin state manipulation can be achieved by resonant microwave radiation. Because of the weak spin-orbital coupling of diamond material, and low concentration of ${ }^{13} \mathrm{C}$, the only spin-carrying isotope of carbon with natural abundance $1.1 \%$, electron spin of NV centers in diamond has very long coherence time $(\sim \mathrm{ms})$ even at room temperature [34]. All these properties mentioned above make $\mathrm{NV}$ centers excellent candidate for quantum logic elements for quantum information processing.

In addition to the applications in quantum information, $\mathrm{NV}$ centers are widely used as solid-state ultra sensitive magnetic field sensor. Again, because of the long coherence time, a tiny change of the magnetic field can be monitored by measuring its effect on the spin dynamics of NV centers. Recent experiments demonstrated high sensitivity $\sim \mathrm{nT} / \sqrt{\mathrm{Hz}}$ at atomic scale resolution [35, 36]. Furthermore, recent research shows that, not only magnetic fields, other types of signal (e.g., electric fields, temperature, and strain etc.) which can be converted to magnetic signal can be also precisely measured by NV center spins. This makes NV centers very amazing multifunctional atomic-scale sensors.

There are two methods to interface the NV centers and the mechanical oscillators. The first one is based on strong magnetic field gradient to couple the mechanical oscillators with NV centers [29]. The second one requires the strain induced effective electric field to mix phonon mode with NV centers electron spins [37]. The strong coupling may reach in both of the methods. Once the strong coupling regime reaches, we may cooling the mechanical oscillator with coherent excitation exchange between spin and motion degrees. We can generate arbitrary superposition states of mechanical oscillator. We may realize phonon laser and squeezing in the system [37, 38]. The system can also be used for quantum in- 
formation processing [30], as it can interface many different degree of freedom.

To increase the coherence time of mechanical oscillator, we can optimize the design and choose the materials with high mechanical $Q$, such as $\mathrm{Si}_{3} \mathrm{~N}_{4}$ with $Q$ around $10^{6}$ [39]. However, the best way is optically trapping the nanodiamond in vacuum [22,40-48]. The mechanical $Q$ factor in this system could be as high as $10^{10}$, which is comparable to the trapped ions. It is possible to cool the trapped nanoparticle from room temperature [49]. Another advantage is that the strong coupling between the oscillation and the spin can be realized with a modest magnetic field gradient around $10^{5}$ $\mathrm{T} / \mathrm{m}$. It is also possible to couple the rotation degree of freedom to the spins. Besides, the trapping frequency can be easily tuned, even completely turned off, which makes the time-of-flight measurement possible [50].

In an optical trap, the motion of nano-particle can be cooled to mK by feedback without cavity [41, 42]. Ground state may reach by means of cavity sideband cooling [43],44], or by exchanging excitations between phonon and spin degrees [29,50,51]. Even at thermal states, matter wave interference could be observed in this systems [50, 52, 53]. By detecting the coherence of NV center spin, the ultra-sensitive mass spectrometer is realizable even at room temperature [54].

This review is organized as follows. In Sec. 2] we will review the methods and applications of strong coupling between mechanical oscillator and NV centers. In Sec. 3, we will review how to observe macroscopic physics and achive ultra-sensitive detection in optically trapped nano-diamond that hosts NV centers. In Sec. 4 we will summarize and discuss the potential developments in the field.

\section{Nanomechanical cantilevers coupled with NV centers}

To couple NV center electron spins with mechanical resonator, we need to apply additional magnetic field gradient, or induce a strain in the lattice of diamond. Here we will briefly discuss these two different methods, and illustrate several applications for both of the methods.

\subsection{Magnetic field gradient induced coupling}

\subsubsection{The setup}

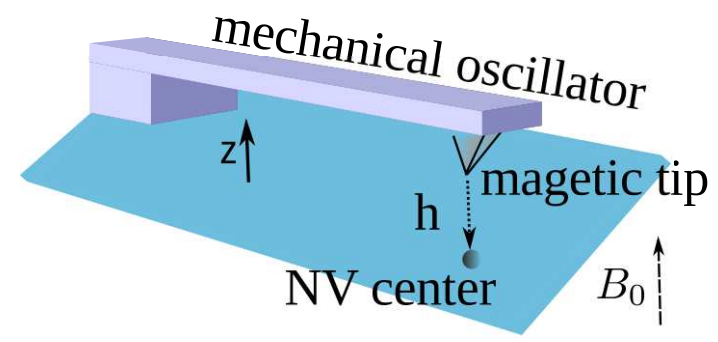

Figure 2 A magnetic tip, which is attached to the end of a cantilever, is placed at a distance $h$ above a single NV center. It creats a strong magnetic gradient near the electronic spin of the NV center. As the spin energy is in proportional to the magnetic field, a strong coupling between the NV center electrons spin and the motion of cantilever is created. Laser field is used for initializing and measuring the spin states. Microwave is used for manipulating the the spin states.

The idea of strong magnetic coupling between a mechanical oscillator and a NV center is shown in Fig. 2] [29]. Here a single NV center electron spin is used for sensing the motion of the mechanical oscillator. It should be noted that the $\mathrm{NV}$ center can also be attached to the end of resonator [31], other than the magnetic tip shown in Fig 2 The frequency of the mechanical oscillator is $\omega_{r}$. The oscillation induces a time-varying magnetic field which causes Zeeman shifts of the electron spin. The single phonon induces frequency shift $\lambda=g_{s} \mu_{b} G_{m} a_{0}$, where $g_{s} \simeq 2, \mu_{B}$ is Bohr magneton, $G_{m}$ is the magnetic field gradient, and $a_{0}=\sqrt{\hbar / 2 m \omega_{r}}$ is the zero field fluctuation for a resonator of mass $m$. We will show that in the current experiments, $\lambda$ can be as high as $100 \mathrm{kHz}$, which is larger than both the spin coherence time $(1 \mathrm{~ms})$, and the damping rate $\gamma=\omega_{r} / Q$ of high $Q$ mechanical resonators.

The Hamiltonian of the system can be written as

$$
H_{S}=H_{N V}+\hbar \omega_{r} a^{\dagger} a+\hbar \lambda\left(a+a^{\dagger}\right) S_{z},
$$

where $H_{N V}$ describes the dynamics of NV center electron spin, $a\left(a^{\dagger}\right)$ is the annihilation(creation) operator of the phonon in mechanical oscillator, and $S_{z}$ is the $z$ component of the spin-1 operator of NV center. We assume that the NV sysmetry axis is also aligned along $z$ direction. The NV center electron spin ground state is an $S=1$ triplet. The three states are labled as $|0\rangle,| \pm 1\rangle$. Without external magnetic field, the levels $| \pm 1\rangle$ are degenerate. The zero field splitting between $|0\rangle$ and $| \pm 1\rangle$ is $\omega_{0} / 2 \pi=2.88 \mathrm{GHz}$. In order to manipulate the levels $| \pm 1\rangle$ individually, we add a static magnetic field $B_{0}$ along $z$ axis to break the degenerate states $| \pm 1\rangle$. The frequencies difference between $|0\rangle$ and $| \pm 1\rangle$ are $\omega_{ \pm 1}=\omega_{0} \pm \mu_{B} B_{0} / \hbar$.

In order to coherent exchange the excitation between mechanical resonator and the spin, we need to add microwaves to drive Rabi oscillations between $|0\rangle$ and $| \pm 1\rangle$. We assume the magnetic field oscillation amplitudes is much less than 
$\hbar \omega_{0} / \mu_{B}$. In rotating wave frame we have

$$
H_{N V}=\sum_{i= \pm 1}-\hbar \Delta_{i}|i\rangle\langle i|+\frac{\hbar \Omega_{i}}{2}(|0\rangle\langle i|+| i\rangle\langle 0|),
$$

where $\Delta_{ \pm 1}$ and $\Omega_{ \pm 1}$ denote the detunings and the Rabi frequencies of the two microwave transitions. For simplicity, we assume that $\Delta_{+1}=\Delta_{-1}=\Delta$ and $\Omega_{+1}=\Omega_{-1}=\Omega$. The eigenstates of $N_{N V}$ are $|d\rangle=(|-1\rangle-|+1\rangle) / \sqrt{2}$, $|g\rangle=\cos \theta|0\rangle-\sin \theta|b\rangle$, and $|e\rangle=\cos \theta|b\rangle+\sin \theta|0\rangle$, where $|d\rangle=(|+1\rangle+|-1\rangle) \sqrt{2}$, and $\tan (2 \theta)=-\sqrt{2} \Omega / \Delta$. The eigenvalues corresponding to are $\omega_{d}=-\Delta$, and $\omega_{e, g}=$ $\left(-\Delta \pm \sqrt{\Delta^{2}+2 \Omega^{2}}\right) / 2$. In the case that $\Delta<0$, the lowest energy state is $|g\rangle$. We adjust the frequency difference between $|d\rangle$ and $|g\rangle \omega_{d g}=\omega_{d}-\omega_{g}$ is equal to the mechanical frequency $\omega_{r}$, and the frequency difference between $|e\rangle$ and $|d\rangle$ $\omega_{e d}=\omega_{e}-\omega_{d}$ is largely detuned with $\omega_{r}$. Under the condition $\left|\omega_{r}-\omega_{e d}\right| \gg \lambda$, we will have effective Jaynes-Cummings Hamiltonian

$$
H_{J C}=\hbar \lambda_{g}|g\rangle\langle e| a^{\dagger}+\text { h.c. }
$$

where $\lambda_{g}=-\lambda \sin \theta$.

In this system, phonon-spin coupling strength $\lambda$ is usually much less than phonon frequency $\omega_{r}$, which is around $10 \mathrm{MHz}$. Therefore, it is impossible to directly generate Schrödinger's cat states in this setup. We will discuss how to realize frequency tunable mechanical oscillator in the next section. By tuning the mechanical frequency less than $\lambda$, the Schorödinger's cat states in macroscopic mechanical resonator can be prepared with spin-dependent force [50].

\subsubsection{Applications as resonator cooling}

With JC Hamiltonian Eq. (3), we can coherently manipulate the mechanical states with the help of spin states if strong coupling condition is fulfilled. The decay of mechanical resonator at temperature $T$ is defined as $\Gamma_{r}=k_{B} T / \hbar Q$. The spin dephasing rate is $\delta \omega_{d g}$. The strong coupling condition is $\lambda_{g}>\Gamma_{r}, \delta \omega_{d g}$. In experiments, we may use $\mathrm{Si}$ cantilever of dimensions $(l, w, t)=(3,0.05,0.05) \mu \mathrm{m}$ with frequency $\omega_{r}=7 \mathrm{MHz}$ and $a_{0}=5 \times 10^{-13} \mathrm{~m}$. A magnetic tip can induce gradient field $G_{m}=10^{7} \mathrm{~T} / \mathrm{m}$ at the distant $h=25 \mathrm{~nm}$. The phonon-spin coupling strength $\lambda=100 \mathrm{kHz}$ is realizable. The mechanical Q can be as high as $10^{5}$ and the heating rate is $\Gamma_{r} / 2 \pi=20 \mathrm{kHz}$. The spin decay can be around $\delta \omega_{g d}=1$ $\mathrm{kHz}$ if we use Carbon-13 purified diamond. Therefore, the strong coupling condition can be fulfilled.

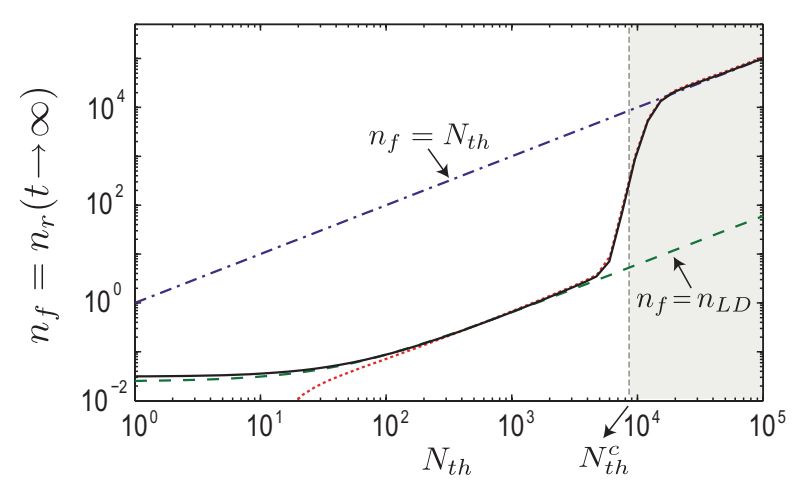

Figure 3 Steady state phonon occupation number $n_{f}$ as a function of environment thermal excitation $N_{t h}$. The value of $N_{t h}^{c}$ denotes the crossover from LD regime to a regime where phonon cooling is strongly suppressed and finally $n_{f}=N_{t h}$. Firgure adopted from Ref. [55]. Copyright (2010) by The American Physical Society.

To manipulate the phonon states of the resonator, the first step is to cool it to the ground state. If the mechanical oscillator frequency is high enough, e.g. $>1 \mathrm{GHz}$, we can use the traditional cryogenic techniques to cool the environment to $10 \mathrm{mK}$, and the resonator is already in its quantum ground state. Usually the resonator frequency is around $\mathrm{MHz}$, we have to use active method to cool it to ground state. In optomechanics, we need cavity mode as an vacuum bath to cool the mechanical mode. In this setup, we don't need cavity, as $\mathrm{NV}$ center can be used as an effective vacuum bath. By using JC Hamiltonian (3) and optical pump technology of NV center spin states, we can cool the resonator to the ground state. The basic idea is as follows. We initialize the NV center spin to $|0\rangle$ by optical pump in the time scale less than $1 \mu \mathrm{s}$. Then we use microwave to prepare the spin state to $|g\rangle$. By turning on the microwave driving we use JC Hamiltonian (3) to exchange transfer excitation from resonator to the NV center spin in the time scale $1 / \lambda_{g} \sim 10 \mu \mathrm{s}$. As last we initialize the $\mathrm{NV}$ center again. By repeating the process, the excitations in resonator are quickly removed. Under the condition $\lambda_{g}>\Gamma_{r}$, the quantum ground state cooling should be possible. If we carefully adjust the detuning of driving microwave and laser, the above procedure can automatically repeated to cool the mechanical resonator [29].

The above cooling method is only valid for low temperature $T<1 \mathrm{~K}$ and mechanical frequency $\omega_{r} \sim 1 \mathrm{MHz}$. For high temperature, the Lamb-Dicke(LD) limit is not fulfilled, the cooling with NV center spins is no longer possible [55], as shown in Fig. 3 When thermal phonon phonon is larger than $N_{t h}^{c}=10^{4}$, the cooling effects quickly disappear. After quantum ground state being prepared, arbitrary superposition states of resonator should be possible. However, to generate the high fidelity phonon states, we need a high $Q$ resonator, which is very difficult for this system. We will discuss this problem in Sec. 3 


\subsubsection{Applications in quantum information processing}

Once mechanical ground state is prepared, the mechanical resonator can be used for quantum information processing [56-58]. By charging the mechanical oscillator, distant coupling between resonators is also possible [30].

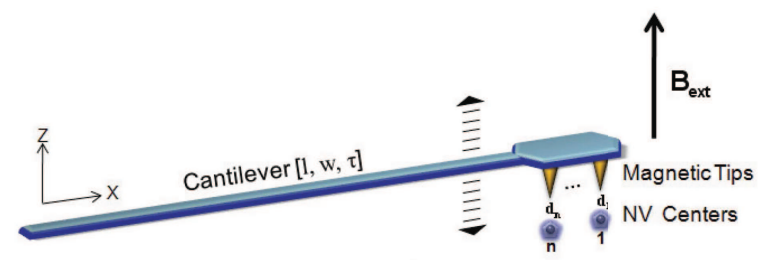

Figure 4 A scheme to entangle multiple NV centers spins that couple with the same mechanical oscillator. (a) An array of evenly spaced magnetic tips are attached at the end of a nanomechanical cantilever, under which are oneto-one correspondent NV centers. Figure adapted from Ref. [57]. Copyright (2010) by The American Physical Society.

In order to entangle distant NV centers, here we need to couple multiple NV centers to the same mechanical oscillator, which is used as a quantum bus. As shown in Fig. 龦, Xu and et al. considered the scheme that an array of a NV centers locate equally distance, above which are one-to-one magnetic tips attached at the end a cantilever. The qubits are encoded in two spins levels 0$\rangle$ and -1$\rangle$. We applied a microwave to globally drive all the NV center spins. We assume that all $\mathrm{NV}$ center spins have the same transition frequency $\omega_{0}$ and the frequency of microwave is on resonant with $\omega_{0}$. We define the new basis for the spins as $| \pm\rangle=(|-1\rangle+|+1\rangle) / \sqrt{2}$. Consider the case that the Rabi frequency of microwave $\Omega$ is comparable to the mechanical frequency $\omega_{r}$, in rotating wave approximation, we have the effective Hamiltonian of the system as

$$
H_{I}=\sum_{j=1}^{n} \frac{\lambda}{2} a|+\rangle_{j}\langle-| \exp [-i \delta(t) d t]+\text { h.c. }
$$

with $\delta(t)=\omega_{r}-\Omega(t)$. The collective Hamiltonian Eq. (4) can be used for generating $\mathrm{W}$ states. As an example, initially we prepare the phonon state to the Fock state $|1\rangle$ with phonon number 1 , and the NV centers to the state $|-\rangle_{1}|-\rangle_{2} \cdots|-\rangle_{n}$. We set the detuning $\delta(t)=0$, the $\mathrm{W}$ state $|W\rangle=$ $\left(|+\rangle_{1}|-\rangle_{2} \cdots|-\rangle_{n}+|-\rangle_{1}|+\rangle_{2} \cdots|-\rangle_{n} \cdots|-\rangle_{1}|-\rangle_{2} \cdots|+\rangle_{n}\right) / \sqrt{n}$ can be generated at the time $t=\pi /(\sqrt{n} \lambda)$.

If the detuning $\delta(t)$ is much larger than coupling strength $\lambda$, it will induce the effective spin-spin coupling between NV centers [56, 59]. In Ref. [56], they only considered two NV centers coupling with the same cantilever. Assume the detuning $\delta_{1}=\delta_{2}=\delta$, to the second order we have the effective Hamiltonian

$$
H_{e f f}=\frac{\hbar \lambda_{g}^{2}}{4 \delta}\left[\left(a^{\dagger} a+\frac{1}{2}\right)\left(\sigma_{1 z}+\sigma_{2 z}\right)-\left(\sigma_{1+} \sigma_{2-}+\sigma_{1-} \sigma_{2+}\right)\right] .
$$

As we discussed in Sec. 2.1.2 the phonon-spin coupling $\hbar \lambda$ could be around $100 \mathrm{kHz}$. In order to fulfill the condition $\delta \gg \lambda_{g}$, we choose $\hbar \delta$ in the order of $1 \mathrm{MHz}$. The spin-spin coupling $\hbar \lambda_{g}^{2} /(4 \delta)$ is in the order of $10 \mathrm{kHz}$, which is larger than both NV center spin decoherence rate and the effective phonon decay rate at $10 \mathrm{mK}$ temperature. Therefore, it is possible to generate the entanglement among NV centers spins in experiments with high fidelity.

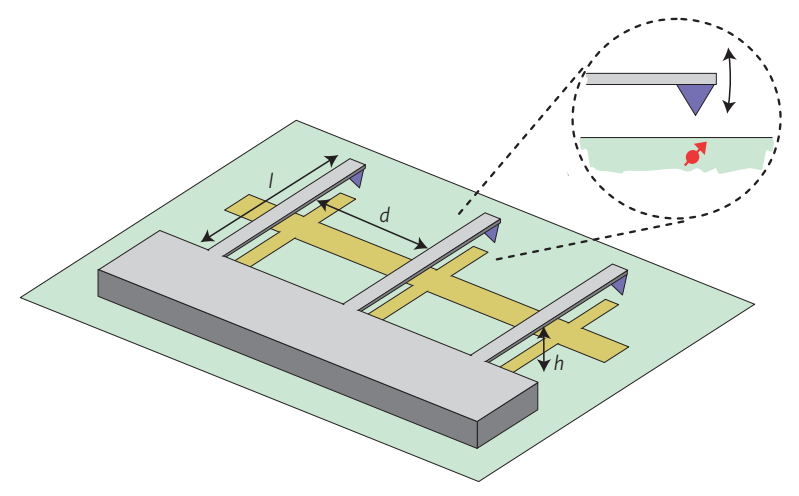

Figure 5 Schematic view of a scalable quantum information processor on an electromechanical quantum bus and NV centers spins as qubits. Figure adapted from Ref. [30]. Copyright (2010) by Nature Publishing Group.

The idea can be extended to realize a scalable quantum information processor [30]. As shown in Fig. [5, the processor consist an array of $N$ nanomechanical resonators, each of which is coupled magnetically to an electronic spin qubit associated with an impurity located in the substrate below. As we discussed in Sec. 2.1.1, the resulting spin-phonon coupling could be as high as $50 \mathrm{kHz}$ under the magnetic gradient $10^{7} \mathrm{~T} / \mathrm{m}$. The long range interaction between distant sites can be established by charging the resonator and capacitively coupling with the nearby wire. The Hamiltonian of the resonators is

$H_{c r}=\sum_{i} \hbar \omega_{r} a_{i}^{\dagger} a_{i}+\frac{\hbar}{2} \sum_{i, j} g_{i j}\left(a_{i}+a_{i}^{\dagger}\right)\left(a_{j}+a_{j}^{\dagger}\right)=\sum_{n} \hbar \omega_{n} a_{n}^{\dagger} a_{n}$

After combining magnetic and electric coupling Hamiltonians, we have the full Hamiltonian of the system,

$$
H_{c r}=H_{s}(t)+\hbar \sum_{n} \hbar \omega_{n} a_{n}^{\dagger} a_{n}+\frac{1}{2} \sum_{i, n} \lambda_{i, n}\left(a_{n}^{\dagger}+a_{n}\right) \sigma_{z}^{i},
$$

where $\left.H_{s} t\right)=\sum_{i} \hbar\left(\delta_{i} \sigma_{z}^{i}+\Omega_{i}(t) \sigma_{x}^{i}\right) / 2, \omega_{n}$ and $a_{n}$ denote frequencies and collective mode operators for phonon eigenmodes. This model is quite similar as the quantum computation proposal for trapped ions systems. However, here the decoherence mechanism and physical implementation are different. Anyway, the idea of using hybrid systems that contain mechanical resonators array and $\mathrm{NV}$ centers for quantum information processing needs to be further investigated in future. 


\subsubsection{Applications in ultra-sensitive measurement}

As the resonator is coupled with NV center spin, we can use NV center as a detector to measure the state of resonator [31,32]. Therefore, the NV center spin can be used as an ultra-sensitive detector for mechanical resonators. Even strong coupling conditions is not fulfilled, we may still detect the Brownian motion of resonator in classical regime.
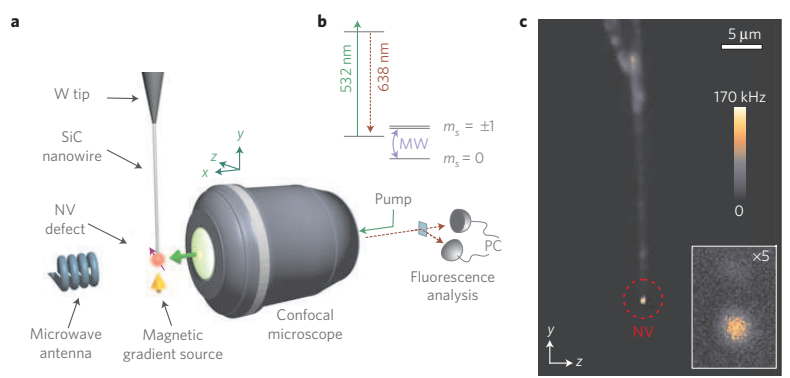

Figure 6 (a) A confocal microscope monitors the fluorescence of a single $\mathrm{NV}$ defect hosted in a diamond nanocrystal placed at the extremity of a SiC nanowire. A microwave antenna is used to manipulate the NV electronic spin, while a micro-fabricated magnetic structure approached in the vicinity of the suspended NV centre generates a strong magnetic field gradient. (b), The electronic levels structure of the NV centres at zero magnetic field. (c), Fluorescence map of the system recorded with the confocal microscope while scanning the objective position. The isolated bright spot circled in red corresponds to the fluorescence of a single NV centre. Inset: zoom on the nanowire extremity. Figure adapted from [31. Copyright (2011) by Nature Publishing Group.

In Ref. [31], it realized the hybrid system that a single nitrogen-vacancy center coupled with a nanomechanical oscillator. A single NV center hosted in a nano-diamond is placed at the extremity of a Sic nanowire. By adding a strong magnetic field gradient to the system, a magnetic coupling between NV center and mechanical oscillator is induced. The motion of nanowire can be probed by reading out a single electron spin of the NV center.

Reference [32] reported the coherent coupling of a mechanical cantilever to the single spin of NV center. The authors used the electronic spin of an NV center to sense the motion of a magnetized cantilever. Although the magnetic field change due to the motion of the cantilever is very small, it can still be monitored by measuring the NV center spin coherence. This was achieve with the help of the spin echo technique, where the spin is flipped by microwave $\pi$ pulses during the evolution. With this technique, the unwanted background noise is filtered out, while the signal to be detected (i.e., the magnetic field signal from the cantilever motion) is magnified. In Ref. [32], the authors successfully demonstrated a motion sensing with precision down to $\sim$ pm under ambient conditions. Later, we will show the spin-echo-based technique is not limit in motion sensing. Indeed, it can be applied in sensing various types of signals [54].

\subsection{Strain induced coupling}

\subsubsection{Model}

In the previous subsection, we summarized the recent works on hybrid systems with a mechanical resonator and a NV center, where a strong magnetic gradient induces spin-phonon coupling. In this section, we will discuss new a mechanism that couples NV center electrons and the the phonon mode in diamond, strain induced spin-phonon coupling, and effective spin-spin interactions between NV centers [37,60]. The spin-spin interactions can induce spin squeezing in NV center ensembles (NVE), which may be used in NVE magnetometry.
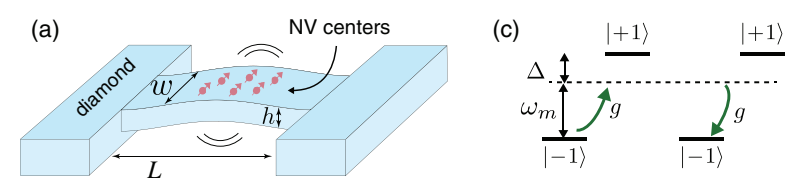

(b)

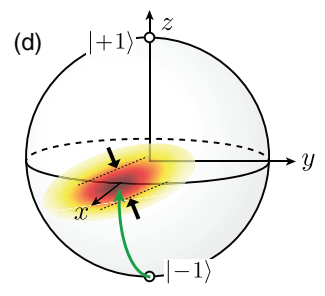

Figure 7 (a) All-diamond doubly clamped mechanical resonator with an embedded NV centers ensemble (NVE). (b) Spin levels and transitions of NV centers electron spins. Local perpendicular strain induced by beam bending mixes the $| \pm 1\rangle$ states. (c) NV centers spins in the two-level subspace $| \pm 1\rangle$ off-resonantly couple with a common mechanical mode. (d) NVE collective spins squeezing. Figure adopted from Ref. 37. Copyright (2013) by The American Physical Society.

As shown in Fig. 7(a), we consider an NVE embedded in a single crystal diamond nanobeam. When beam oscillates, it strains the lattice of diamond, and induces direct coupling between NV center electrons spins and the mechanical mode. Here we write down the Hamiltonian for NV center, in the presence of external electric and magnetic fields $\vec{E}$ and $\vec{B}[37,61]$

$$
\begin{aligned}
H_{\mathrm{NV}}= & \left(D_{0}+d_{\|} E_{z}\right) S_{z}^{2}+\mu_{B} g_{s} \vec{S} \cdot \vec{B} \\
& -d_{\perp}\left[E_{x}\left(S_{x} S_{y}+S_{y} S_{x}\right)+E_{x}\left(S_{x}^{2}-S_{y}^{2}\right)\right],
\end{aligned}
$$

where $D_{0} / 2 \pi=2.88 \mathrm{GHz}$ is the zero field splitting, $g_{s} \simeq 2$, $\mu_{B}$ is the Bohr magneton, and $d_{\|}\left(d_{\perp}\right)$ is the ground state electric dipole moment in the direction parallel (Perpendicular) to the NV axis [62].

As shown in Fig. 7(b), motion of nanoresonator changes the local strain of the NV center, and induces an effective electric field. We are interested to the near resonant coupling between a single motion mode and $| \pm 1\rangle$ transition of the NV center. The Zeeman splitting between $| \pm 1\rangle$ is $\Delta_{B}=g_{s} \mu_{B} B_{z} / \hbar$. The perpendicular component of strain $E_{\perp}$ mixes $| \pm 1\rangle$ states with interaction Hamiltonian $E_{\perp}=E_{0}\left(a+a^{\dagger}\right)$, where $a$ is the 
destruction operator of the resonator mode with frequency $\omega_{m} . E_{0}$ is the zero point motion of the beam induced perpendicular strain. The parallel strain also induces the level shifts between $| \pm 1\rangle$ and $|0\rangle$. However, in the subspace $| \pm 1\rangle$ the parallel strain plays no role in the system, as state $|0 r\rangle$ is not involved. In this subspace, the interaction Hamiltonian of each NV center is

$$
H_{I}=\hbar g\left(a^{\dagger} \sigma_{i}^{-}+a \sigma_{i}^{+}\right)
$$

where $\sigma_{i}^{ \pm}=| \pm 1\rangle\langle\mp 1|$ is Pauli operator for $i$ th NV center and $g$ is the single phonon coupling strength. For NVE, we can define the collective spin operators, $J_{z}=\frac{1}{2} \sum_{i}|1\rangle_{i}\langle 1|-|-1\rangle_{i}\langle-1|$ and $J_{ \pm}=J_{x} \pm i J_{y}=\sum_{i} \sigma_{i}^{ \pm}$. The total Hamiltonian is

$$
H_{T}=\omega_{m} a^{\dagger} a+\Delta_{B} J_{z}+g\left(a^{\dagger} J_{-}+a J_{+}\right) \text {. }
$$

Here for simplicity, we assume that coupling $g$ is uniform for all NV centers. The interaction between NV centers is also neglected as we assume that they are far apart from each other.

The coupling strength $g$ can be estimated with the follow equation [37]

$$
\frac{g}{2 \pi} \approx 180\left(\frac{\hbar}{L^{3} w \sqrt{\rho E}}\right)^{1 / 2},
$$

where $\rho$ is the mass density and $E$ is the Young's modulus of diamond. Up to now, we only achieved the single phonon coupling strength $g \sim 0.25 \mathrm{~Hz}$ in a diamond resonator [60]. Theoretically, we may get $g \sim 1 \mathrm{kHz}$ in future. For a beam of dimensions $(L, w, h)=(1,0.1,0.1) \mu \mathrm{m}$ we got $\omega_{m} / 2 \pi \sim 1$ $\mathrm{GHz}$ and coupling $g / 2 \pi \sim 1 \mathrm{kHz}$. We define the single spin cooperativity $\eta=g^{2} T_{2} /\left(\gamma \bar{n}_{t h}\right)$, where $\gamma=\omega_{m} / Q$ is the mechanical decay rate and $T_{2}$ is the spin dephasing time, and $\bar{n}_{t h}$ is the thermal equilibrium phonon number for resonator. It is found that $\eta$ could be as high as 0.8 for $T=4 \mathrm{~K}, T_{2}=10 \mathrm{~ms}$, and $Q=10^{6}$. To get $\eta>1$, we need to further decrease the dimension of the diamond resonator, or decrease the temperature $T$. Similarly as Sec., the Hamiltonian can also be used for cooling the mechanical oscillator if the strong coupling condition is fulfilled [38].

\subsubsection{Spin squeezing}

Similarly as we discussed in Sec. 2.1.3 [56, 59], in large detuning limit that $g \ll \Delta=\Delta_{B}-\omega_{m}$, we can adiabatically eliminate the mechanical mode $a$, and get effective spin-spin interactions [37]. The effective Hamiltonian is

$$
H_{e f f}=\omega_{m} a^{\dagger} a+\left(\Delta_{B}+\lambda a^{\dagger}\right) J_{z}+\frac{\lambda}{2} J_{+} J_{-},
$$

where $\lambda=2 g^{2} / \Delta$. The Hamiltonian Eq. (12) can be used for generating spin squeezing state. To generate the spin squeezed state, we initialized the NVE to coherent spin state $\left|\psi_{0}\right\rangle$ along $x$ axis which satisfies $J_{x}\left|\psi_{0}\right\rangle=J\left|\psi_{0}\right\rangle$. It has equal transverse variances $\left\langle J_{x}^{2}\right\rangle=\left\langle J_{x}^{2}\right\rangle=J / 2$. The interaction term
$J_{+} J_{-}=\mathbf{J}^{2}-J_{x}^{2}+J_{z}$, where total angular momentum $J$ is conserved and $J_{z}^{2}$ induces spin variance squeezing in one direction, as shown in Fig. 7(d). The squeezing can be qualified by the parameter

$$
\xi^{2}=\frac{2 J\left\langle\Delta J_{\min }^{2}\right\rangle}{\left\langle J_{x}\right\rangle^{2}}
$$

where $\left\langle\Delta J_{\min }^{2}\right\rangle=\frac{1}{2}\left(V_{+}-\sqrt{v_{-}^{2}+V_{y z}^{2}}\right)$ is the minimum spin uncertainty with $V_{ \pm}=\left\langle J_{y}^{2} \pm J_{z}^{2}\right\rangle$ and $V_{y z}=\left\langle J_{y} J_{z}+J_{z} J_{y}\right\rangle / 2$. If we prepare the spin squeezed state with $\xi^{2}<1$, it has a direct applications for magnetometry of NVE below the projection noise ilmit [63].
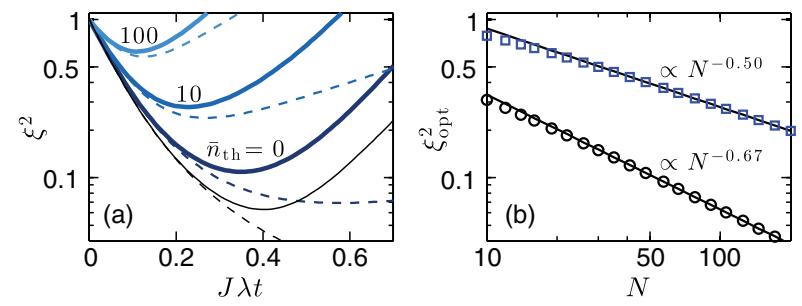

Figure 8 (a) Spin squeezing parameter versus scaled precession time with $N=100$ spins. Thick blue (gray) lines show the calculated squeezing parameter for $T_{2}=10 \mathrm{~ms}$ and values of $\bar{n}_{\mathrm{th}}$ as shown. For each curve, the squeezing is optimized. Dashed lines are calculated from the linearized equations for the spin operator averages. (b) Optimal squeezing versus number of spins. Lower (upper) line shows power law fit for $\bar{n}_{\mathrm{th}}=1(10)$ and $T_{2}=1(0.01) \mathrm{s}$ . Other parameters in both plots are $\omega_{m}=1 \mathrm{GHz}, g / 2 \pi=1 \mathrm{kHz}, Q=10^{6}$. Figure adopted from Ref. [37]. Copyright (2013) by The American Physical Society.

In Fig 8(a), the squeezing parameter verse time is plotted for an ensemble of $N=100$ spins and several $\bar{n}_{\text {th }}$. The decoherence of spin and mechanical decay is also considered by solving master equation [37]. We plot the scaling of the squeezing parameter with $J$ for small decoherence in Fig. 8 b). It was found the scalling is $\xi_{\text {opt }}^{2} \sim J^{-2 / 3}$.

\section{Optically trapped nano-diamond that hosts NV centers}

As we stated in the introduction, one of the key factors affecting macroscopic Schrödinger's cat state is the mechanical Q factor. In this section we will focus on the optically trapped nanodiamond system, whose mechanical Q factor is not related the the material, but the pressure of the vacuum. For the current technology, we can have $Q$ has high as $10^{10}$. Therefore, the life time of Schrödinger's cat state can be as long as millisecond. 


\subsection{Scheme and Fock States Preparation}

(a)

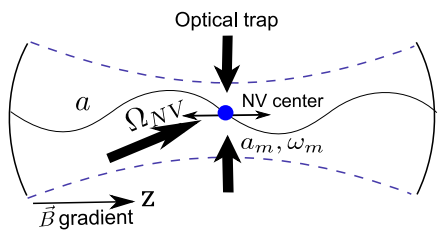

(b)

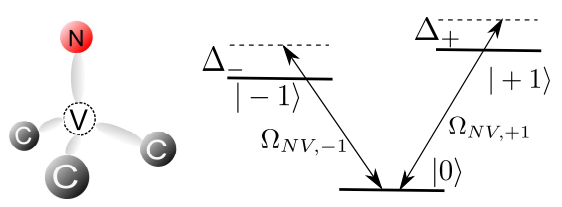

Figure 9 (a) A nanodiamond with a NV center is optically trapped in vacuum with spin-mechanical coupling enabled through a nearby magnetic tip and opto-mechanical coupling through a cavity around. (b) The atomic structure (left) and the level diagram (right) in the ground state manifold for a NV center in the nanodiamond. Figure adopted from [50]. Copyright (2013) by The American Physical Society.

As shown in Fig. 97, we consider a nano-diamond trapped by an optical tweezers in a high-Q cavity in vacuum. Therefore, the mechanical motion of the nanodiamond couples with the cavity mode. The trap is located in a place that the cavity mode has the maximum gradient. Near the NV center, there is a magnet tip, which induces a strong magnetic field gradient. The magnetic gradient couples the mechanical motion and the electron spin. There is also a microwave source to control the spin of the NV center inside the nanodiamond. As the nanodiamond is optically trapped in the vacuum, the coherence time of the mechanical mode for its center-of-mass motion is long [50]. The frequency of the optical trap can be quickly tuned through control of the laser intensity. This feature is important as we can cool the mechanical mode to the ground state in a strong trap and prepare large quantum superposition states of the nanodiamond in a weak trap by a quench of the trap frequency.

Once the resonator cooled down, we can generate many non-classical states, such as Fock states. The NV spin is initially set to the state $|0\rangle$, which is decoupled from the mechanical mode during the cooling. Initialization and single shot detection of the NV spin have been well accomplished experimentally [64]. We assume that the NV center is at a position with zero magnetic field and a large field gradient. We apply a microwave drive with the Hamiltonian $H_{\text {drive }}=\hbar\left(\Omega_{\mathrm{NV},+1} e^{i \omega_{l+} t}|0\rangle\left\langle+1\left|+\Omega_{\mathrm{NV},-1} e^{i \omega_{l-} t}\right| 0\right\rangle\langle-1|+\right.$ h.c. $) / 2$ and set the Rabi frequency $\Omega_{\mathrm{NV}, \pm 1}=\Omega_{\mathrm{NV}}$ and the detuning $\Delta_{ \pm} \equiv \omega_{l \pm}-\omega_{ \pm 1}=\Delta$. With $\Delta \gg\left|\Omega_{\mathrm{NV}}\right|$, we adiabatically eliminate the level $|0\rangle$ and get the following effective Hamiltonian

$$
H_{e}=\hbar \omega_{m} a_{m}^{\dagger} a_{m}+\hbar \Omega \sigma_{z}+\hbar \lambda\left(\sigma_{+}+\sigma_{-}\right)\left(a_{m}+a_{m}^{\dagger}\right)
$$

where $\Omega=\left|\Omega_{\mathrm{NV}}\right|^{2} / 4 \Delta, \sigma_{z}=|+\rangle\langle+|-|-\rangle\left\langle-\left|, \sigma_{+}=\right|+\right\rangle\langle-|$, $\sigma_{-}=|-\rangle\langle+|$, and we have defined the new basis states $|+\rangle=(|+1\rangle+|-1\rangle) / \sqrt{2},|-\rangle=(|+1\rangle-|-1\rangle) / \sqrt{2}$. In the limit $\lambda \ll \omega_{m}$, we set $\Omega=\omega_{m} / 2$ and use the rotating wave approximation to get an effective interaction Hamiltonian between the mechanical mode and the NV center spin, with the form

$$
H_{J C}=\hbar \lambda \sigma_{+} a_{m}+h . c . .
$$

This represents the standard Jaynes-Cummings $(\mathrm{J}-\mathrm{C})$ coupling Hamiltonian. Similarly, if we set $\Omega=-\omega_{m} / 2$, the anti $\mathrm{J}-\mathrm{C}$ Hamiltonian can be realized with

$$
H_{a J C}=\hbar \lambda \sigma_{+} a_{m}^{\dagger}+\text { h.c.. }
$$

Arbitrary Fock states and their superpositions can be prepared with a combination of J-C and anti J-C coupling Hamiltonians. For example, to generate the Fock state $|2\rangle_{m}$, we initialize the state to $|+\rangle|0\rangle_{m}$, turn on the J-C coupling for a duration $t_{1}=\pi /(2 \lambda)$ to get $|-\rangle|1\rangle_{m}$, and then turn on the anti $\mathrm{J}-\mathrm{C}$ coupling for a duration $t_{2}=t_{1} / \sqrt{2}$ to get $|+\rangle|2\rangle_{m}$. The Fock state with arbitrary phonon number $n_{m}$ can be generated by repeating the above two basic steps, and the interaction time is $t_{i}=t_{1} / \sqrt{i}$ for the $i$ th step. Superpositions of different Fock states can also be generated. For instance, if we initialize the state to $\left(c_{0}|+\rangle+c_{1}|-\rangle\right) \otimes|0\rangle_{m} / \sqrt{2}$ through a microwave with arbitrary coefficients $c_{0}, c_{1}$, and turn on the $\mathrm{J}-\mathrm{C}$ coupling for a duration $t_{1}$, we get the superposition state $|-\rangle \otimes\left(c_{1}|0\rangle_{m}+i c_{0}|1\rangle_{m}\right) / \sqrt{2}$. Using the optical cavity, the Fock state $\left|n_{m}\right\rangle_{m}$ of mechanical mode can also be mapped to the corresponding Fock state of the output light field [43].

The effective QND Hamiltonian for the spin-phonon coupling takes the form

$$
H_{Q N D}=\hbar \chi \sigma_{z} a_{m}^{\dagger} a_{m},
$$

with $\chi=4 \Omega \lambda^{2} /\left(4 \Omega^{2}-\omega_{m}^{2}\right)$ when the detuning ||$\Omega\left|-\omega_{m} / 2\right| \gg$ $\lambda$. The Hamiltonian $H_{Q N D}$ can be used for a quantum nondemolition measurement(QND) measurement of the phonon number: we prepare the NV center spin in a superposition state $\left.|+\rangle+e^{i \phi}|-\rangle\right) / \sqrt{2}$, and the phase $\phi$ evolves by $\phi(t)=$ $\phi_{0}+2 \chi n_{m} t$, where $n_{m}=a_{m}^{\dagger} a_{m}$ denotes the phonon number. Through a measurement of the phase change, one can detect the phonon number.

Let us estimate the typical parameters. A large magnetic field gradient can be generated by moving the nanodiamond close to a magnetic tip. Though magnetic gradient up to $10^{7} \mathrm{~T} / \mathrm{m}$ has been realized $[65,66$, here we take the gradient $G=10^{5} \mathrm{~T} / \mathrm{m}$, which is much less than the one used in Sec. 2.1.2 [29]. We get the coupling $\lambda \simeq 2 \pi \times 52 \mathrm{kHz}$ for a nanodiamond with the diameter $d=30 \mathrm{~nm}$ in an optical trap with a trapping frequency $\omega_{m}=2 \pi \times 0.5 \mathrm{MHz}$. The QND detection rate $2|\chi| \sim 2 \pi \times 25 \mathrm{kHz}$ with the detuning ||$\Omega\left|-\omega_{m} / 2\right| \sim 5 \lambda$. The NV electron spin dephasing time over $1.8 \mathrm{~ms}$ has been observed at room temperature [34], which is long compared with the Fock state preparation time $1 / \lambda$ and the detection time $1 /(2|\chi|)$.

\subsection{Macrocopic quantum superpositions}

To prepare spatial quantum superposition state, we need to generate quantum superposition of the nanodiamond at dis- 
tinct locations. To detect the superposition state, we need to do interference experiment, either with matter wave [50] or with the spin [52].

\subsubsection{Matter wave interference}

Without the microwave driving, the spin-mechanical coupling Hamiltonian takes the form

$$
H=\hbar \omega_{m} a_{m}^{\dagger} a_{m}+\hbar \lambda S_{z}\left(a_{m}+a_{m}^{\dagger}\right)
$$

The mechanical mode is initialized to the vacuum state $|0\rangle_{m}$ (or a Fock state $\left|n_{m}\right\rangle_{m}$ ) in a strong trap with the trapping frequency $\omega_{m 0}$ and the $\mathrm{NV}$ center spin is prepared in the state $|0\rangle$. Although the ground state cooling is most effective in a strong trap, to generate large spatial separation of the wave packets it is better to first lower the trap frequency by tuning the laser intensity for the optical trap. While it is possible to lower the trap frequency through an adiabatic sweep to keep the phonon state unchanged, a more effective way is to use a nonadiabatic state-preserving sweep [67], which allows arbitrarily short sweeping time. We denote $\left|n_{m}\right\rangle_{m 1}$ as the mechanical state in the lower frequency $\omega_{m 1}$. We then apply an impulsive microwave pulse to suddenly change the NV spin to the state $(|+1\rangle+|-1\rangle) / \sqrt{2}$ and simultaneously decrease the trap frequency to $\omega_{m 2} \leqslant \omega_{m 1}$. The evolution of the system state under the Hamiltonian (15) then automatically split the wave packet for the center-of-mass motion of the nanodiamond. The splitting attains the maximum at time $T_{2} / 2=\pi / \omega_{m 2}$, where the maximum distance of the two wave packets in the superposition state is $D_{m}=8 \lambda a_{2} / \omega_{m 2}=4 g_{s} \mu_{B} G /\left(m \omega_{m 2}^{2}\right)$, where $a_{2}=\sqrt{\hbar / 2 m \omega_{m 2}}$. At this moment, the system state is

$$
\left|\Psi_{S}\right\rangle=\left(|+1\rangle\left|D_{m} / 2\right\rangle_{n_{m}}+|-1\rangle\left|-D_{m} / 2\right\rangle_{n_{m}}\right) / \sqrt{2},
$$

where $\left| \pm D_{m} / 2\right\rangle_{n_{m}} \equiv(-1)^{a_{m}^{\dagger} a_{m}} e^{ \pm D_{m}\left(a_{m}^{\dagger}-a_{m}\right) / 4 a_{2}}\left|n_{m}\right\rangle_{1}$ is the displaced Fock state (or coherent states when $n_{m}=0$ ). This is just the entangled spatial superposition state.

The maximum distance $D_{m}$ is plotted in Fig. 10 versus trap frequency, magnetic field gradient, and diameter $d$ of the nanodiamond, and superposition states with separation $D_{m}$ comparable to or larger than the diameter $d$ is achievable under realistic experimental conditions.
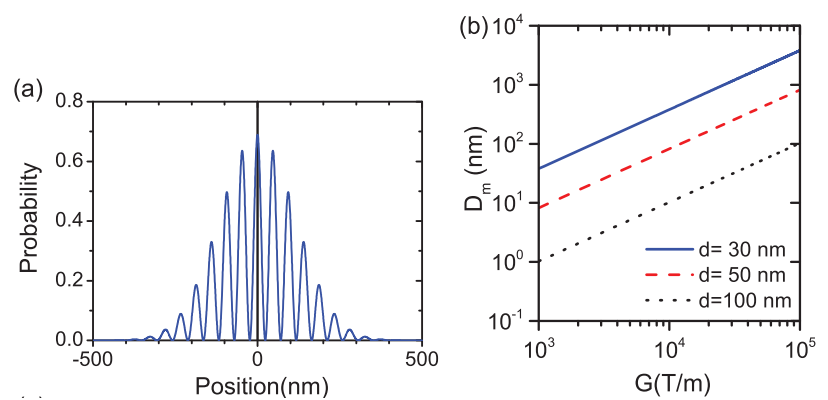

Figure 10 (a) Spatial interference patterns for a $30 \mathrm{~nm}$ nano-diamond af ter $10 \mathrm{~ms}$ of free expansion. The nano-diamond is initially prepared in the vacuum state $|0\rangle_{m}$ or the 1 -phonon state $|1\rangle_{m}$ of a $20 \mathrm{kHz}$ trap. The magnetic gradient is $3 \times 10^{4} \mathrm{~T} / \mathrm{m}$. Before the trap is turned off, the center of mass of the nano-diamond is prepared in $\left|\psi_{+}\right\rangle_{0}$. (b) Maximum spatial separation $D_{m}$ as a function of the magnetic gradient $G$ when the trapping frequency is $1 \mathrm{kHz}$. Macroscopic superposition states with separation larger than the size of the particle can be achieved with a moderate magnetic gradient. Figure adopted from [50]. Copyright (2013) by The American Physical Society.

To transform the entangled cat state $\left|\Psi_{S}\right\rangle$ to the standard cat state $\left|\psi_{ \pm}\right\rangle_{n_{m}} \equiv\left(\left|D_{m} / 2\right\rangle_{n_{m}} \pm\left|-D_{m} / 2\right\rangle_{n_{m}}\right) / \sqrt{2}$, we need to apply a disentangling operation to conditionally flip the $\mathrm{NV}$ spin using displacement of the diamond as the control qubit. This can be achieved as different displacements of the wavepacket induce relative energy shifts of the spin levels due to the applied magnetic field gradient [50]. To detect spatial superposition state, we can turn off the optical trap and let the spatial wave function freely evolve for some time $t$. The split wave packets will interference just like the Young's double slit experiment. The period of the interference pattern is $\Delta z=2 \pi \hbar t /\left(m D_{m}\right)$. As an estimation of typical parameters, we take $\omega_{m 1}=\omega_{m 2}=2 \pi \times 20 \mathrm{kHz}, d=30 \mathrm{~nm}$, and magnetic field gradient $3 \times 10^{4} \mathrm{~T} / \mathrm{m}$. The spin-phonon coupling rate $\lambda \simeq 2 \pi \times 77 \mathrm{kHz}$ and the maximum distance $D_{m} \simeq 31 a_{2}$. The preparing time of superposition state is about $25 \mu \mathrm{s}$, which is much less than the coherence time of the NV spin. For the time of flight measurement after turnoff of the trap, we see the interference pattern with a period of $47 \mathrm{~nm}$ after $t=10 \mathrm{~ms}$, as shown in Fig. 10, which is large enough to be spatially resolved [41,42].

\subsubsection{Ramsey interference}

The challenging of matter wave interference proposal is that the mass and velocity various for an ensemble of nanodiamond [52]. In order to overcome this challenging, Ref. [52] proposed to used Ramsay interferometry to observe the interference. With Ramsay interence, we can verify the matter wave superpositions, as well as the quantum contextuality for macroscopic quantum systems [53]. The basic setup of their poposal is the same as Fig. 9. Here we assume that an angle $\theta$ between the vertical and the $z$ axis of the system. The Hamiltonian of the system is

$$
H=\hbar D S_{z}^{2}+\hbar \omega_{r} a_{m}^{\dagger} a_{m}-2 \hbar\left(\lambda S_{z}-\Delta \lambda\right)\left(a_{m}+a_{m}^{\dagger}\right)
$$


where $D=2.87 \mathrm{GHz}$ is the zero field splitting, and $\Delta \lambda=$ $\frac{1}{2} m g \cos \theta \sqrt{\frac{\hbar}{2 m \omega_{r}}}$. Let's denote $\mu=2\left(S_{z} \lambda-\Delta \lambda\right) / \hbar \omega_{r}$. The initial state of resonator is a coherent state $|\beta\rangle$. The spin dependent time evolution of the state is $\left|\beta\left(t, S_{z}\right)\right\rangle\left|S_{z}\right\rangle$, where $S_{z}=0, \pm 1$. We get that

$$
\left.\beta\left(t, S_{z}\right)\right\rangle=e^{-i\left(D-\omega_{r} \mu^{2}\right) t+i \mu^{2} \sin \left(\omega_{r} t\right)}\left|(\beta-\mu) e^{-i \omega_{r} t}+\mu\right\rangle .
$$

A striking feature of this evolution is that the oscillator returns to its original coherent state $\beta$ for any $\beta$ and $S_{z}$ at time $t_{0}=2 \pi / \omega_{r}$.

As an example, we consider a initial separable state $|\beta\rangle\left|S_{z}\right\rangle$. By using a strong microwave pulse $H_{m w}=\hbar \Omega(|+1\rangle\langle 0|+|-$ $1\rangle\langle 0|+$ h.c., we can change the state to $|\Psi(0)\rangle=\beta\rangle(|+1\rangle+$ $|-1\rangle) / \sqrt{2}$. The state after an oscillation period $t_{0}=2 \pi / \omega_{r}$ becomes

$$
\left|\Psi\left(t_{0}\right)\right\rangle=|\beta\rangle\left(\frac{|+1\rangle+e^{i \Delta \phi_{g}}|-1\rangle}{\sqrt{2}}\right) .
$$

where we have $\Delta \phi_{g}=16 \lambda \Delta \lambda t_{0} /\left(\hbar^{2} \omega_{r}\right)$. We apply microwave Hamiltonian $H_{m w}$ again to transfer the spin population to $|0\rangle$. After time $t_{p}=\pi /(2 \sqrt{2} \Omega)$, we get

$$
P_{0}=\cos ^{2}\left(\frac{\Delta \phi_{g}}{2}\right)
$$

which can be used to measure the relative phase $\Delta \phi_{g}$. The detection of $\Delta \phi_{g}$ is an evidence for the matter wave superposition state. Besides, as the phase $\Delta \phi_{g}$ is not dependent to the initial state $\beta$, it is possible to be observed when initial state is thermal.

\subsection{Applications as mass spectrometer}

As mentioned above, one of the most attractive features of the system of optically levitated particles is its high mechanical quality factor. The trapped particle can coherently oscillator many times (e.g., $10^{10}$ times) before its mechanical energy is damping out. When this kind of almost-perfect mechanical oscillator couples to a long-live quantum object, i.e. NV center electron spin, fantastic quantum phenomena and novel application can be expected.

In Ref. [54], we studied the quantum dynamics of a coupled system of a quantum oscillator and a single spin, where the spin is under dynamical decoupling control. Dynamical decoupling control, as an extended version of spin echo technique, has been proved to be an efficient method to prolong the spin coherence time. In Ref. [54] we predicted that, under dynamical decoupling control and coupled to a high-quality mechanical oscillator, the spin coherence will exhibit a series of periodic peaks, forming a comb structure (called timecomb in Ref. [54]). The peak width is calculated as

$$
\Delta_{q^{*}} \equiv \frac{2 \sqrt{2}}{\gamma_{q^{*}}}=\frac{T_{0}}{N \Lambda \sqrt{2 n_{\mathrm{th}}+1}} .
$$
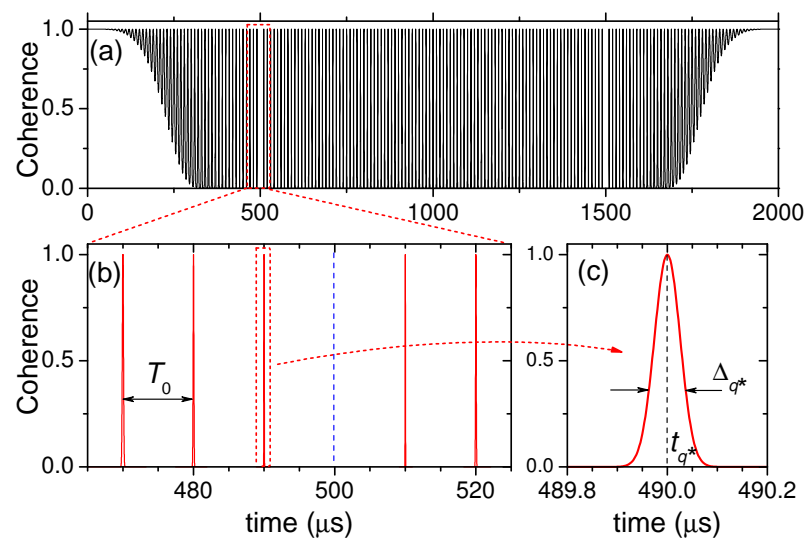

Figure 11 (a) The time-comb structure of qubit coherence under 100-pulse CPMG control. For $\omega_{r} t \gg 1$, the comb period is synchronized with the oscillator period $T_{0}=2 \pi / \omega_{r}$. (b) Close-up of the coherence peaks. A missing peak at $\omega_{r} t=N \pi$ is indicated by the blue dashed line. The peak width is decreasing when getting close to the missing one. (c) Close-up of the narrowest coherence peak, which is centered at $t_{q^{*}}$ with width $\Delta_{q^{*}}$ (see text). The parameters used in this figure are oscillator frequency $100 \mathrm{kHz}$, coupling strengt $\lambda=0.0001 \omega_{r}$, temperature $T=10 \mathrm{~K}$ and 100-pulse CPMG control.

Two features are notable in the above equation. First, the peak width is inversely proportional to the square root of the thermal occupation number $n_{\text {th }}$ of the mechanical oscillator, which implies narrower peaks in higher temperature. Second, the peak width is inversely proportional to the control pulse number $N$. Since the control pulse number $N$ is related to the total coherent evolution time, and the later can be regarded to a kind of resource in high-precision sensing, we expected that improving the dynamical decoupling control pulse number can improve the measurement sensitivity.

Based on the time-comb structure, we propose an ultra-sensitive mass spectrometer using the coupled qubitoscillator system. Since the peak position in the time axis is synchronized with oscillator period, we propose to infer the oscillator period (and its mass assuming a constant spring coefficient) by measuring the peak position. Obviously according to the error propagation formula, the narrower the peak is, the better sensitivity we will have. The two features mentioned above make the proposed mass spectrometer different to those based on traditional measurement principles. As the peak will be narrower in higher temperature, we propose that the mass spectrometer will have a counterintuitive temperature dependence of the sensitivity (i.e., higher temperature helps improve the sensitivity). This result makes the roomtemperature application of ultra-sensitive mass spectrometer possible. The second feature, as we showed in Ref. [54], implies an improved scaling of the measurement sensitivity to the measurement resource. The scaling relation goes beyond the shot-noise scaling and Heisenberg scaling in the quantum metrology theory. The underlying physics which brings about this improved scaling is under further studying. 


\subsection{Experimental progresses}

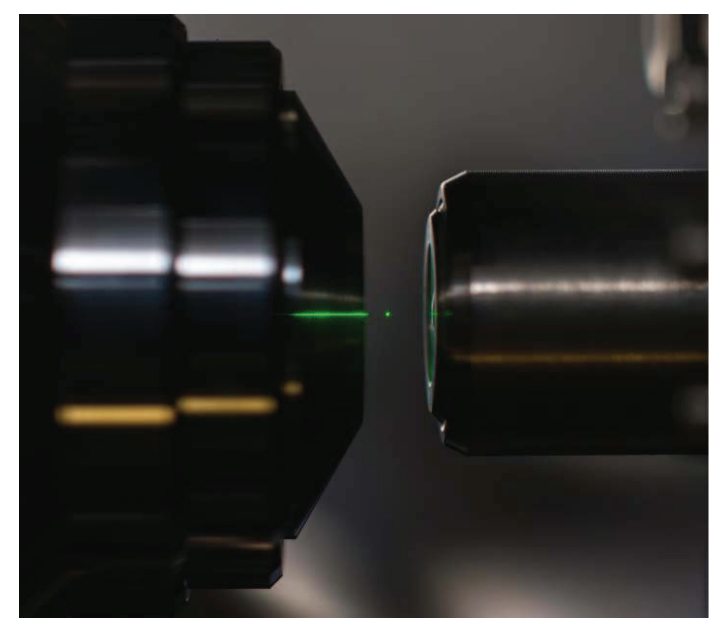

Figure 12 A photograph of a $\sim 100$ diameter nanodiamond which is levitated by a optical tweezer $(1064 \mathrm{~nm})$. The green color is from an optical pump $532 \mathrm{~nm}$ laser. The trapping lens is on the left, and the lens on the right is used to collimate the light exiting the trap. Figure adopted from [46]. Image credit: J. Adam Fenster, University of Rochester.

Dielectric nanoparticles have been trapped and cooled optically, as shown in Ref. [41, 42, 44]. Following the similar method, trapping nanodiamond in atmosphere pressure has been realized experimentally in 2013 [51]. The experimental setup is shown in Fig. 12 They found the evidence of NV photoluminescence from a nanodiamond in a free-space optical dipole trap. The photoluminescence rates are decreased with increasing trap laser power. The neutral charge state $\left(\mathrm{NV}^{0}\right)$ can be suppressed by continuous-wave trap.

The charged nanodiamond was also levitated in a linear quadrupole ion trap in air under ambient conditions [68]. An electric trap has no trapping laser that quenches fluorescence emission. The surface charge has effects on the emission rates of fluorescence spectrum of NV center. However, there is no difference in the spectral properties was found in the experiment. Up to now, there is no report on trapping nanodiamond in vacuum. This is the first problem that should be overcome in future experiment.

\section{Conclusion and Outlook}

In this paper we gave a brief review on the field of (opto)nano-mechanical resonator coupling with $\mathrm{NV}$ centers electrons spins. We discussed magnetic and strain induced phonon-spin coupling mechanisms. We discussed how to cool the resonator to the ground state and how to manipulate the phonon non-classical states. We also discuss how to realize quantum information processing in this system. We reviewed the progresses in optically trapped nano-diamond with building in NV centers. We discussed how to observe macroscopic quantum interference in the system. We also discussed how to used the system as an ultra-sensitive mass spectrometer in room temperature. Finally, we briefly summarized the experimental progresses.

Here we discuss some new directions and ideas in the field. Up to now, we only consider the first order magnetic gradient induced coupling. It would be interest to study the second order gradient induced spin-resonator coupling. This coupling mechanism may have some new applications and features. For example, it may be used to entangle NV centers with different transition frequencies. It may also be used for cooling mechanical resonator which is out of Lamb-Dicke regime.

Another new direction is to study the coupling between rotation degree of freedom of trapped nano-diamond to the internal NV centers electron spins. Geometric phase, even non-Abelian geometric phase may be induced by mechanical rotation [69,70]. The rotation may also be cooled and manipulated by NV centers.

It would be interesting to investigate how to cool down the internal temperature of the diamond which hosts the NV centers. As we know, the decay rate of the NV centers electron spins is in verse proportional to the temperature. Only under the low temperature $T<10 \mathrm{~K}$, the decay rate can be decreased to around $\mathrm{Hz}$. By considering the coupling between $\mathrm{NV}$ center electron spins and the phonons in the diamond, it would be actively cooled down the temperature of a nanodiamond trapped with an optical tweezers [71,72]. In this way, we may extend the lifetime of NV centers electron spins around second even at room temperature environment.

Before conclusion, we note that the proposals and schemes discussed in this review should also be applied in other similar systems, such as hybrid mechanical resonator with quantum dots [73], ${ }^{2} 8 \mathrm{Si}$ nanoparticles with donor spins [74], or nanocrystals doped with rare-earth ions [75].

ZQY is funded by the NBRPC (973 Program) 2011CBA00300 (2011CBA00302), NNSFC NO. 11105136, NO. 61435007. NZ is supported by NKBRP (973 Program) 2014CB848700 and NNSFC No. 11374032 and No. 11121403. TL acknowledges the support from Purdue University through the startup fund.

1 The Nobel Prize in Physics 2012, URL www.nobelprize.org

2 Wieman C E, Pritchard D E, Wineland D J. Atom cooling, trapping, and quantum manipulation. Rev Mod Phys, 1999, 71: S253.

3 Wineland D J. Nobel Lecture: Superposition, entanglement, and raising Schrödingers cat. Rev Mod Phys, 2013, 85: 1103.

4 Monroe C, Meekhof D M, King B E, et al. A Schrödinger cat superposition state of an atom. Science, 1996, 272(5265): 1131-1136.

5 Einstein A. On the development of our views concerning the nature and constitution of radiation. Phys. Z, 1909, 10: 817.

6 Braginski V B, Manukin A B. Ponderomotive effects of electromagnetic radiation. Sov. Phys.JJETP, 1967, 25: 653.

7 Ashkin A. Acceleration and trapping of particles by radiation pressure. Phys Rev Lett, 1970, 24: 156.

8 Ashkin A, Dziedzic J M. Optical levitation by radiation pressure. App Phys Lett, 1971, 19: 283-285. 
9 Aspelmeyer M, Meystre P, Schwab K. Quantum optomechanics. Physics Today, 2012, 65: 29-35.

10 Aspelmeyer M, Kippenberg T J, Marquardt F. Cavity optomechanics. arXiv preprint, 2013, arXiv:1303.0733.

11 Liu Y C; Hu Y W, Wong C W, et a. Review of cavity optomechanical cooling. Chin. Phys. B, 2013, 22: 114213.

12 OConnell A D, Hofheinz M, Ansmann M, et al. Quantum ground state and single-phonon control of a mechanical resonator. Nature, 2010, 464: 697-703.

13 Teufel J D, Donner T, Li D, et al. Sideband cooling of micromechanical motion to the quantum ground state. Nature, 2011, 475: 359-363.

14 Chan J, Alegre T P M, Safavi-Naeini A H, et al. Laser cooling of a nanomechanical oscillator into its quantum ground state. Nature, 2011, 478: 89-92.

15 Verhagen E, Deléglise S, Weis S, et al. Quantum-coherent coupling of a mechanical oscillator to an optical cavity mode. Nature, 2012, 482(7383): 63-67.

16 Weis S, Riviére R, Delglise S, et al. Optomechanically induced transparency. Science, 2010, 330: 1520-1523.

17 Purdy T P, Yu P L, Peterson R W, et al. Strong optomechanical squeezing of light. Phys Rev X, 2013, 3: 031012.

18 Wang Y D, Clerk A A. Using Interference for High Fidelity Quantum State Transfer in Optomechanics. Phys Rev Lett, 2012, 108: 153603.

19 Andrews R W, Peterson R W, Purdy T P, et al. Bidirectional and efficient conversion between microwave and optical light. Nat Phys, 2014, 10: 321.

20 Yin Z, Yang W L, Sun L, et al. Quantum network of superconducting qubits through opto-mechanical interface. arXiv preprint arXiv:1407.4938, 2014.

21 Romero-Isart O, Pflanzer A C, Blaser F, et al. Large quantum superpositions and interference of massive nanometer-sized objects. Phys Review Lett, 2011, 107: 020405.

22 Romero-Isart O, Juan M L, Quidant R, et al. Toward quantum superposition of living organisms. New J Phys, 2010, 12: 033015.

23 Penrose R. On gravity's role in quantum state reduction. General relativity and gravitation, 1996, 28: 581-600.

24 Ghirardi G C, Rimini A, Weber T. Unified dynamics for microscopic and macroscopic systems. Phys Rev D, 1986, 34: 470.

25 Ghirardi G C, Pearle P, Rimini A. Markov processes in Hilbert space and continuous spontaneous localization of systems of identical particles. Phys Rev A, 1990, 42: 78.

26 Wilson-Rae I, Zoller P, Imamoḡlu A. Laser cooling of a nanomechanical resonator mode to its quantum ground state. Phys Rev Lett, 2004, 92: 075507.

27 Bennett S D, Cockins L, Miyahara Y, et al. Strong electromechanical coupling of an atomic force microscope cantilever to a quantum dot. Phys Rev Lett, 2010, 104: 017203.

28 Hammerer K, Wallquist M, Genes C, et al. Strong coupling of a mechanical oscillator and a single atom. Phys Rev Lett, 2009, 103: 063005.

29 Rabl P, Cappellaro P, Dutt M V G, et al. Strong magnetic coupling between an electronic spin qubit and a mechanical resonator. Phys Rev B, 2009, 79: 041302.

30 Rabl P, Kolkowitz S J, Koppens F H L, et al. A quantum spin transducer based on nanoelectromechanical resonator arrays. Nat Phys, 2010, 6: 602-608. $\mathrm{p}$

31 Arcizet O, Jacques V, Siria A, et al. A single nitrogen-vacancy defect coupled to a nanomechanical oscillator. Nature Physics, 2011, 7: 879883.

32 Kolkowitz S, Jayich A C B, Unterreithmeier Q P, et al. Coherent sensing of a mechanical resonator with a single-spin qubit. Science, 2012, 335: 1603-1606.

33 Bar-Gill N, Pham L M, Belthangady C, et al. Suppression of spin-bath dynamics for improved coherence of multi-spin-qubit systems. Nature communications, 2012, 3: 858 .

34 Balasubramanian G, Neumann P, Twitchen D, et al. Ultralong spin coherence time in isotopically engineered diamond. Nature materials, 2009, 8: 383-387.

35 Zhao N, Honert J, Schmid B, et al. Sensing single remote nuclear spins. Nature Nanotechnology, 2012, 7: 657-662.

36 Shi F, Kong X, Wang P, et al. Sensing and atomic-scale structure analysis of single nuclear-spin clusters in diamond. Nature Physics, 2014, 10: 21-25.

37 Bennett S D, Yao N Y, Otterbach J, et al. Phonon-induced spin-spin interactions in diamond nanostructures: application to spin squeezing. Phys Rev Lett, 2013, 110: 156402.

38 Kepesidis K V, Bennett S D, Portolan S, et al. Phonon cooling and lasing with nitrogen-vacancy centers in diamond. Phy Rev B, 2013, 88(6): 064105 .

39 Zwickl B M, Shanks W E, Jayich A M, et al. High quality mechanical and optical properties of commercial silicon nitride membranes. Applied Physics Letters, 2008, 92: 103125.

40 Chang D E, Regal C A, Papp S B, et al. Cavity opto-mechanics using an optically levitated nanosphere. PNAS, 2010, 107: 1005-1010.

41 Li T, Kheifets S, Raizen M G. Millikelvin cooling of an optically trapped microsphere in vacuum. Nat Phys, 2011, 7: 527-530.

42 Gieseler J, Deutsch B, Quidant R, et al. Subkelvin parametric feedback cooling of a laser-trapped nanoparticle. Phys Rev Lett, 2012, 109: 103603.

43 Yin Z, Li T, Feng M. Three-dimensional cooling and detection of a nanosphere with a single cavity. Phys Rev A, 2011, 83: 013816.

44 Kiesel N, Blaser F, Delić U, et al. Cavity cooling of an optically levitated submicron particle. PNAS, 2013, 110: 14180-14185.

45 Yin Z, Geraci A A, Li T. Optomechanics of Levitated Dielectric Particles. Int J Mod Phys B, 2013, 27: 1330018.

46 Neukirch L P, Vamivakas A N. Nano-optomechanics with optically levitated nanoparticles. Contemporary Physics, 2014 (ahead-of-print): 115.

47 Nie W, Lan Y, Li Y, et al. Dynamics of a levitated nanosphere by optomechanical coupling and Casimir interaction. Physical Review A, 2013, 88: 063849.

48 Nie W J, Lan Y H, Li Y, et al. Generating large steady-state optomechanical entanglement by the action of Casimir force[J]. Science China Physics, Mechanics \& Astronomy, 2014, 57: 2276-2284.

49 Liu Y C, Liu R S, Dong C H, et al. Cooling mechanical resonators to quantum ground state from room temperature, 2014, arXiv:1406.7359.

50 Yin Z, Li T, Zhang X, et al. Large quantum superpositions of a levitated nanodiamond through spin-optomechanical coupling. Phys Rev A, 2013, 88: 033614.

51 Neukirch L P, Gieseler J, Quidant R, et al. Observation of nitrogen vacancy photoluminescence from an optically levitated nanodiamond. Optics letters, 2013, 38: 2976-2979.

52 Scala M, Kim M S, Morley G W, et al. Matter-wave interferometry of a levitated thermal nano-oscillator induced and probed by a spin. Phys Rev Lett, 2013, 111: 180403.

53 Asadian A, Brukner C, Rabl P. Probing Macroscopic Realism via Ramsey Correlation Measurements. Phys Rev Lett, 2014, 112: 190402.

54 Zhao N, Yin Z. Room-temperature ultra-sensitive mass spectrometer via dynamic decoupling. Phys Rev A, 2013, 90:042118.

55 Rabl P. Cooling of mechanical motion with a two-level system: The high-temperature regime. Phys Rev B, 2010, 82: 165320.

56 Zhou L, Wei L F, Gao M, et al. Strong coupling between two distant electronic spins via a nanomechanical resonator. Phys Rev A, 2010, 81: 042323.

57 Xu Z Y, Hu Y M, Yang W L, et al. Deterministically entangling distant nitrogen-vacancy centers by a nanomechanical cantilever. Phys Rev A, 
2009, 80: 022335.

58 Chen Q, Xu Z, Feng M. Entanglement generation of nitrogen-vacancy centers via coupling to nanometer-sized resonators and a superconducting interference device. Phys Rev A, 2010, 82: 014302.

59 Zheng S B, Guo G C. Efficient scheme for two-atom entanglement and quantum information processing in cavity QED. Phys Rev Lett, 2000, 85: 2392 .

60 Teissier J, Barfuss A, Appel P, et al. Strain Coupling of a NitrogenVacancy Center Spin to a Diamond Mechanical Oscillator. Phys Rev Lett, 2014, 113: 020503.

61 Doherty M W, Dolde F, Fedder H, et al. Theory of the ground-state spin of the NV-center in diamond. Phys Rev B, 2012, 85: 205203.

62 Dolde F, Fedder H, Doherty M W, et al. Electric-field sensing using single diamond spins. Nature Physics, 2011, 7: 459-463.

63 Ma J, Wang X, Sun C P, et al. Quantum spin squeezing. Physics Reports, 2011, 509: 89-165.

64 Robledo L, Childress L, Bernien H, et al. High-fidelity projective readout of a solid-state spin quantum register. Nature, 2011, 477: 574-578.

65 Tsang C, Bonhote C, Dai Q, et al. Head challenges for perpendicular recording at high areal density. Magnetics, IEEE Transactions on, 2006, 42: 145-150.

66 Mamin H J, Poggio M, Degen C L, et al. Nuclear magnetic resonance imaging with 90-nm resolution. Nature nanotechnology, 2007, 2: 301306.

67 Chen X, Ruschhaupt A, Schmidt S, et al. Fast optimal frictionless atom cooling in harmonic traps: Shortcut to adiabaticity. Phys Rev Lett, 2010, 104: 063002.

68 Kuhlicke A, Schell A W, Zoll J, et al. Nitrogen vacancy center fluorescence from a submicron diamond cluster levitated in a linear quadrupole ion trap. Applied Physics Letters, 2014, 105: 073101.

69 Maclaurin D, Doherty M W, Hollenberg L C L, et al. Measurable quantum geometric phase from a rotating single spin. Phys Rev Lett, 2012, 108: 240403.

70 Kowarsky M A, Hollenberg L C L, and Martin A M. Non-Abelian geometric phase in the diamond nitrogen-vacancy center. Phys Rev A, 2014 90:042116.

71 Seletskiy D V, Melgaard S D, Bigotta S, et al. Laser cooling of solids to cryogenic temperatures. Nature Photonics, 2010, 4: 161-164.

72 Zhang J, Li D, Chen R, et al. Laser cooling of a semiconductor by 40 kelvin. Nature, 2013, 493: 504-508.

73 Bell D M, Howder C R, Johnson R C, et al. Single CdSe/ZnS Nanocrystals in an Ion Trap: Charge and Mass Determination and Photophysics Evolution with Changing Mass, Charge, and Temperature. ACS nano 2014, 8: 2387-2398.

74 Steger M, Saeedi K, Thewalt M L W, et al. Quantum information storage for over $180 \mathrm{~s}$ using donor spins in a $28 \mathrm{Si}$ semiconductor vacuum. Science, 2012, 336: 1280-1283.

75 Afzelius M, Chaneliére T, Cone R L, et al. Photon-echo quantum memory in solid state systems. Laser \& Photonics Reviews, 2010, 4: 244267. 